\title{
JOINT DIRECTION-OF-ARRIVAL AND ORDER ESTIMATION IN COMPRESSED SENSING USING ANGLES BETWEEN SUBSPACES
}

\author{
Mads Graesboll Christensen \\ Dept. of Arch., Design and Media Technology \\ Aalborg University, Denmark \\ mgcecreate.aau.dk
}

\author{
Jesper Kjar Nielsen \\ Dept. of Electronic Systems \\ Aalborg University, Denmark \\ jkn@es.aau.dk
}

\begin{abstract}
In this paper, we consider the problem of joint direction-of-arrival and order estimation in array processing with compressed sensing. In particular, we show how to solve these problems jointly using a subspace approach based on the notion of angles between subspaces. In the process, we also discuss the conditions on the measurement matrix and demonstrate how to implement the estimator algorithm efficiently when using compressed sensing. Our simulation results show that it is indeed possible to solve these problems and that good performance can be obtained, although the use of compressed sensing does have an impact on the performance of the estimator.
\end{abstract}

Index Terms - Direction of arrival estimation, spectral analysis, frequency estimation, array signal processing

\section{INTRODUCTION}

A classical problem in array signal processing is that of determining the direction-of-arrival (DOA) of sources impinging on the array, and many methods have been proposed throughout the history, including such prominent work as [1-3]. We are here also interested in this problem, but in a new context, namely that of compressed sensing $[4,5]$, wherein measurements are formed as random linear combinations of the sensor inputs. In this context, the parameter estimation and signal reconstruction problems are most often dealt with by solving convex problems, typically equality constrained 1norm minimization problems, and such approaches have also been applied to array signal processing, e.g., [6-8]. In both DOA estimation and compressed sensing, the number of sources, i.e., the model order, is often assumed known, or, is assumed to have been found in some other way. The number of sources may of course vary over time, and the question is, however, how to deal with this both in DOA estimation and in compressed sensing, since the design of an appropriate measurement matrix requires that the number of sources is known, i.e., that the level of spatial sparsity is known a priori.

The problem under consideration can be formally defined as follows. Let $y_{k}(n)$ be the observed signal at time $n$ for sensor $k$ of a uniform linear array (ULA), and let $K$ be the total number of sensors and $N$ the total number of snapshots. For $L$ narrowband sources (the model order) and complex spatial noise $\mathbf{e}(n)$ impinging on the array, the spatial signal model can be expressed as

$$
\mathbf{y}(n)=\mathbf{\Phi} \mathbf{A} \mathbf{x}(n)+\mathbf{\Phi} \mathbf{e}(n)
$$

where $\mathbf{x}(n)$ contains the individual signals of the sources impinging on the array and $\mathbf{y}(n)$ contains $y_{k}(n)$ for $k=1, \ldots, K$. The noise is here assumed to be colored, i.e., $\mathrm{E}\left\{\mathbf{e}(n) \mathbf{e}^{H}(n)\right\}=\mathbf{Q}$ with $\mathbf{Q}$ being the noise covariance matrix, $\mathrm{E}\{\cdot\}$ the expectation operator and $(\cdot)^{H}$ the conjugate transpose. We here assume that $\mathbf{Q}$ is known or estimated in some other way and that it is invertible. Moreover, $\mathbf{A} \in \mathbb{C}^{K \times L}$ is a Vandermonde matrix containing the steering vectors of the $L<K$ incoherent sources, with unknown spatial frequencies $\left\{\omega_{l}\right\}$, defined as $\mathbf{A}=\left[\begin{array}{lll}\mathbf{a}\left(\omega_{1}\right) & \cdots & \mathbf{a}\left(\omega_{L}\right)\end{array}\right]$ where $\mathbf{a}\left(\omega_{l}\right)=$ $\left[\begin{array}{lll}1 e^{-j \omega_{l}} & \cdots & e^{-j \omega_{l}(K-1)}\end{array}\right]^{T}$ is the steering vector of source $l$. We then seek to find the spatial frequencies $\left\{\omega_{l}\right\}$ and the number of sources $L$. The spatial frequencies are related to the DOAs as $\omega_{l}=$ $\Omega_{l} d \sin \theta_{l} / c$ with $\Omega_{l}$ being the center frequency of the $l$ th source, $\theta_{l}$ its DOA, $d$ the sensor spacing, and $c$ the propagation velocity. Assuming that the spatial frequencies are distinct, the columns of $\mathbf{A}$ are linearly independent. The matrix $\boldsymbol{\Phi} \in \mathbb{R}^{M \times K}$ with $M \leq K$ is the so-called measurement or sensing matrix of compressed sensing (see, e.g., $[4,5]$ ), which here operates across the array exploiting spatial sparsity. We will return to the matter of how to choose $M$ later. This matrix is constructed as a realization of a random process but is assumed known and constant over the $N$ snapshots.

In this paper, we present a subspace-based approach for determining the direction-of-arrivals as well as the number of sources, i.e., the model order. The method is based on a modified covariance matrix model that takes the presence of compressed sensing into account. At this point, it should be stressed that we are not here arguing for the relevance of using compressed sensing in this context, but rather investigating how the associated problems can be solved in a consistent manner (for some applications of compressed sensing, we refer the reader to [5]). The proposed method is based on the concept of angles between subspaces (see, e.g., $[9,10]$ ), which has recently been shown to be applicable to the problem of model order estimation [11].

The remainder of this paper is organized as follows: In Section 2 we develop the covariance matrix for signals of the form (1) and discuss the implications of using compressed sensing on the model. We then proceed to present the proposed joint DOA and order estimator in Section 3 and present some results in Section 4. Finally, we conclude our work in Section 5.

\section{MODIFIED COVARIANCE MATRIX MODEL}

We will now proceed to derive the modified covariance matrix for the compressed sensing scenario. The $M \times M$ covariance matrix of the observed signal is then

$$
\mathbf{R}=\mathrm{E}\left\{\mathbf{y}(n) \mathbf{y}^{H}(n)\right\}=\mathbf{\Phi} \mathbf{A} \mathbf{P} \mathbf{A}^{H} \boldsymbol{\Phi}^{T}+\boldsymbol{\Phi} \mathbf{Q} \boldsymbol{\Phi}^{T} .
$$

Assuming that the signals of the individual sources in the vector $\mathbf{x}(n)$ are independent and zero-mean, the matrix $\mathbf{P}$ is diagonal and contains the expected power of the individual sources. 
From (2), it can be observed that the measurement matrix generally changes the covariance matrix of the noise, rendering even white noise colored, and this must be addressed before we proceed. We will here do this by introducing a pre-whitener that takes the presence of colored noise and compressed sensing into account as follows. Let $\mathbf{C}$ be the Cholesky factor such that

$$
\left(\boldsymbol{\Phi} \mathbf{Q} \boldsymbol{\Phi}^{T}\right)^{-1}=\mathbf{C}^{H} \mathbf{C}
$$

with $\mathbf{C}$ being a square, lower triangular matrix. We note that $\mathbf{\Phi} \mathbf{Q} \Phi^{T}$ is square, positive definite and has full rank and thus invertible with probability close to one. Note that the Cholesky factor is generally complex due to $\mathbf{Q}$ being complex. Then, by multiplying $\mathbf{y}(n)$ by $\mathbf{C}$, we obtain a pre-whitened signal whose covariance matrix is given by

$$
\widetilde{\mathbf{R}}=\mathbf{C} \boldsymbol{\Phi} \mathbf{A P A}^{H} \boldsymbol{\Phi}^{T} \mathbf{C}^{H}+\mathbf{I} .
$$

Note that the noise variance can vary and be unknown without affecting the derivations that follow. Let $\mathbf{U} \boldsymbol{\Lambda} \mathbf{U}^{H}$ be the eigenvalue decomposition (EVD) of $\widetilde{\mathbf{R}}$. Then, $\mathbf{U}$ contains the $M$ orthonormal eigenvectors $\mathbf{u}_{m}$, and $\boldsymbol{\Lambda}$ is a diagonal matrix containing the corresponding eigenvalues, $\lambda_{m}$, with $\lambda_{1} \geq \ldots \geq \lambda_{M}$. Let $\mathbf{S}$ be formed from the eigenvectors corresponding to the $L$ most significant eigenvalues, the range $\mathcal{R}(\cdot)$ of which we refer to as the signal subspace. Similarly, let $\mathbf{G}$ be formed from the eigenvectors corresponding to the $M-L$ least significant eigenvalues, i.e., $\mathbf{G}=\left[\begin{array}{ccc}\mathbf{u}_{L+1} & \cdots & \mathbf{u}_{M}\end{array}\right]$, and $\mathcal{R}(\mathbf{G})$ is referred to as the noise subspace. It can then be shown that the columns of $\mathbf{C \Phi A}$ span the same space as the columns of $\mathbf{S}$, and that $\mathbf{C \Phi A}$ therefore also must be orthogonal to $\mathbf{G}$, i.e.,

$$
\mathbf{A}^{H} \boldsymbol{\Phi}^{T} \mathbf{C}^{H} \mathbf{G} \triangleq \mathbf{A}^{H} \boldsymbol{\Xi}=\mathbf{0},
$$

or, equivalently, A should be orthogonal to $\boldsymbol{\Xi}$. This result is an extension of the basic result used in the MUSIC algorithm as originally proposed in [1], here modified to account for compressed sensing. In practice, $\mathbf{G}$ is of course unknown and an estimate can be obtained from the EVD of the sample covariance matrix. From the above, it can then be seen that to estimate $\mathbf{G}$, we must require that $M>L$. Additionally, we observe that it is required that $\operatorname{rank}(\boldsymbol{\Phi A})=L$, which essentially means that we must construct a measurement matrix $\boldsymbol{\Phi}$ that, regardless of what the spatial frequencies $\left\{\omega_{l}\right\}$ are, must have rows that capture or are likely to capture the column space of $\mathbf{A}$, i.e., $\mathcal{R}(\mathbf{A}) \in \mathcal{R}\left(\boldsymbol{\Phi}^{T}\right)$. This is essentially also what the so-called restricted isometry property (RIP) of compressed sensing says, and some ways of constructing matrices (and choosing $M$ ) that obey this have been proposed in the literature [12]. Until recently [13], these conditions were only shown to hold for what has been referred to as incoherent dictionaries, but such conditions are in direct contradiction with the physics of the considered problem as the individual spatial angles can occur on a continuum of values, corresponding to a highly coherent dictionary. The question still remains, however, how to choose $M$ when $L$ is unknown. We here propose to simply put an upper bound on it and choose $M$ accordingly (see, e.g., [5, 13] for details), and hence facilitate estimating the exact $L$ number of sources as long as it is lower than this bound. Regarding the number of snapshots $N$, it must be at least as high as the number of sources, $L$, to allow identification of the signal subspace and its orthogonal complement, i.e., $N \geq L$. Note that the covariance matrix need not be full rank for this approach to work.

On a related note, the issue of the coherence of the dictionary is also closely related to an advantage that the proposed method holds over methods like basis pursuit [14], since such methods are inherently restricted to finite dictionaries, while the proposed method can (finite precision effects aside) find underlying continuous parameters, corresponding to an infinite and highly coherent dictionary.

\section{MEASURING ORTHOGONALITY}

The question is now how to measure the orthogonality between the two matrices $\mathbf{A}$ and $\boldsymbol{\Xi}$. In answering this question, we will turn to the notion of angles between subspaces in linear algebra. Let $\Pi_{\Xi}$ be the projection matrix for the subspace $\mathcal{R}(\boldsymbol{\Xi})$ and $\boldsymbol{\Pi}_{A}$ the projection matrix for the subspace $\mathcal{R}(\mathbf{A})$. The principal angles between the two subspaces are defined recursively as (see, e.g., [9])

$$
\begin{aligned}
\cos \left(\theta_{k}\right) & =\max _{\mathbf{y} \in \mathbb{C}^{M}} \max _{\mathbf{z} \in \mathbb{C}^{M}} \frac{\mathbf{y}^{H} \boldsymbol{\Pi}_{A} \boldsymbol{\Pi}_{\Xi \mathbf{z}}}{\|\mathbf{y}\|_{2}\|\mathbf{z}\|_{2}} \\
& \triangleq \mathbf{y}_{k}^{H} \boldsymbol{\Pi}_{A} \boldsymbol{\Pi}_{\Xi} \mathbf{z}_{k}=\xi_{k},
\end{aligned}
$$

for $k=1, \ldots, \kappa$ and orthogonal vectors $\mathbf{y}^{H} \mathbf{y}_{i}=0$ and $\mathbf{z}^{H} \mathbf{z}_{i}=0$ for $i=1, \ldots, k-1$. Furthermore, $\kappa$ is the minimal dimension of the two subspaces, i.e., $\kappa=\min \{L, K-L\}$. As can be seen, $\left\{\xi_{k}\right\}$ are the singular values of the matrix product $\Pi_{A} \Pi_{\Xi}$. As was shown in [11], a convenient and accurate scalar measure of the angles is the average over cosine to the angles squared, i.e.,

$$
\frac{1}{\kappa} \sum_{k=1}^{\kappa} \cos ^{2}\left(\theta_{k}\right)=\frac{1}{\kappa}\left\|\boldsymbol{\Pi}_{A} \boldsymbol{\Pi}_{\Xi}\right\|_{F}^{2} .
$$

For computing this, we need to determine the two projection matrices. For $\boldsymbol{\Xi}$ this is not problem as it has to be calculated only once for each set of snapshots and candidate $L$. It is, however, more problematic for $\mathbf{A}$, as it depends on all the DOAs- this is also the reason that $\mathbf{\Phi}$ and $\mathbf{C}$ are multiplied onto $\mathbf{G}$ rather than $\mathbf{A}$. Noting that the columns in $\mathbf{A}$ are asymptotically orthogonal, i.e., $\lim _{K \rightarrow \infty} K \mathbf{\Pi}_{A}=\lim _{K \rightarrow \infty} K \mathbf{A}\left(\mathbf{A}^{H} \mathbf{A}\right)^{-1} \mathbf{A}^{H}=\mathbf{A} \mathbf{A}^{H}$, we see that the projection matrix can be simplified significantly. Let $\boldsymbol{\Gamma}$ be an orthogonal basis for $\mathcal{R}(\boldsymbol{\Xi})$. Then its projection matrix is given by $\boldsymbol{\Pi}_{\Xi}=\boldsymbol{\Gamma} \boldsymbol{\Gamma}^{H}$ and (8) can be expressed as

$$
\frac{1}{\kappa} \sum_{k=1}^{\kappa} \cos ^{2}\left(\theta_{k}\right)=\frac{1}{\kappa}\left\|\mathbf{A}^{H} \boldsymbol{\Gamma}\right\|_{F}^{2} .
$$

This measure can now be used to determine the model order $L$ as well as the spatial frequencies $\left\{\omega_{l}\right\}$ as the parameters that combine to minimize the average angle between the two subspaces, i.e., (see [11] for details)

$$
\left(\hat{L},\left\{\hat{\omega}_{l}\right\}\right)=\arg \min _{L} \frac{1}{\kappa} \sum_{l=1}^{L} \min _{\omega_{l}}\left\|\mathbf{a}^{H}\left(\omega_{l}\right) \boldsymbol{\Gamma}\right\|_{F}^{2},
$$

which follows from the additivity of the Frobenius norm over the columns of $\mathbf{A}$. Note that both $\mathbf{A}$ and $\boldsymbol{\Gamma}$ depend on $L$ while only $\mathbf{A}$ depends on $\left\{\omega_{l}\right\}$. Hence, (10) is not equivalent to simply estimating the order by identifying peaks in the pseudo-spectrum. (10) is a practical measure as the minimization over $\left\{\omega_{l}\right\}$ is decoupled into $L$ minimizations over one nonlinear parameter. Moreover, the inner products involved in the computation of $\mathbf{a}^{H}\left(\omega_{l}\right) \boldsymbol{\Gamma}$ can be efficiently computed using FFTs, or, alternatively, using standard polynomial rooting methods. We note that it can be seen from the definition of angles between subspaces that the measure used in the original MUSIC algorithm is only correct when both the involved matrices consist of orthogonal columns. 


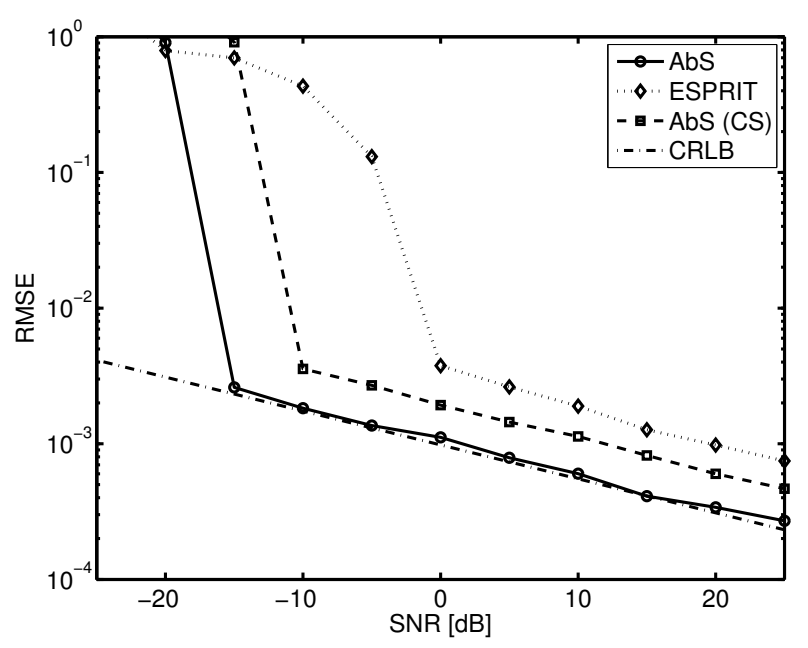

Fig. 1. RMSE as a function of the SNR.

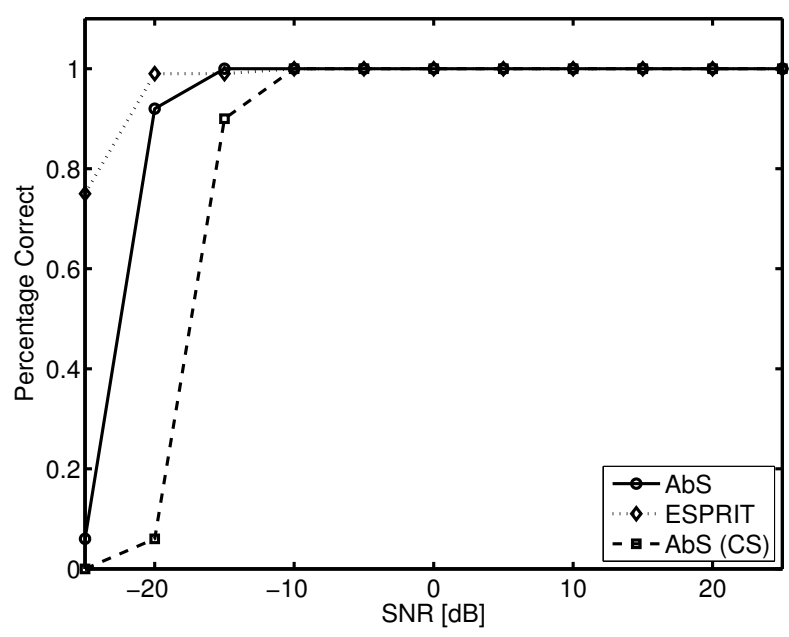

Fig. 2. Percentage of correctly estimated model orders as a function of the SNR.

\section{RESULTS}

We will now report some experimental results in the form of root mean square estimation errors (RMSEs) for the spatial frequencies as well as the percentage of correctly estimated model orders. The results were obtained using Monte Carlo simulations with 100 runs for each data point. The dependencies of the RMSE on various factors have been investigated by varying the signal-to-noise ratio (SNR), the number of measurements $M$ retained in the compressed sensing, and the number of snapshots $N$ in separate experiments. As reference, the Cramér-Rao lower bound (CRLB) is reported as well, and we compare to another method capable of joint DOA and order estimation, namely ESPRIT [2] extended to order estimation as proposed in [15]. Measurement matrices generated as realizations of a Gaussian i.i.d. process with $M=4 L$ as has been reported to work well in practice [5] are used, except in the experiment in which $M$ is varied. These were randomized in each Monte Carlo run and were then also compared to the performance without compressed sensing. That is, by setting the measurement matrix equal to the identity matrix, the proposed method reduces to MUSIC, except that it also determines the model order. In the figures to follow, we refer to the case with compressed sensing as CS and the proposed method based on angles between subspaces as AbS. For all the experiments, additive, white, Gaussian noise was used along with $L=5$ narrowband sources, impinging on the array from different angles. For simplicity in the experiment (and for retaining the same SNR for all sources), these sources were generated having distinct spatial frequencies $\left\{\omega_{l}\right\}$, namely $0.7966,2.2467,3.1414,4.4963$, and 6.2727, identical power and i.i.d. uniformly distributed phases. Except when otherwise stated, an SNR of $10 \mathrm{~dB}$ was used, along with $K=50$ and $N=50$. In Figures 1-6, the RMSE and the percentage of correctly estimated model orders are shown as functions of the various parameters. First of all, it can be observed that the MUSIC method performs close to the CRLB when compressed sensing is not used. This is, however, not the case when compressed sensing is used, as a gap can be observed. Interestingly, we observe that this gap is approximately equal to the ratio between $K$ and $M$, i.e., the performance obtained with compressed sensing is identical to what one would have obtained by simply using $M$ sensors instead of $K$. It can also be observed that the proposed method determines the correct model order, except under adverse conditions with very low SNRs and low $M$, and, as $M$ is increased, its performance approaches the CRLB. Note that several of the methods exhibit identical performance over some intervals in Figures 4 and 6, for which reason the curves fall on top of each other. An interesting observation from these experiments is that the threshold behavior changes with the use of compressed sensing, meaning that the estimator breaks down earlier than without compressed sensing. Another observation is that even with compressed sensing, the proposed method outperforms the ESPRIT algorithm in terms of RMSE.

\section{CONCLUSION}

In this paper, we have considered the problem of jointly determining the direction-of-arrivals and the number of sources jointly in arrays employing compressed sensing. We have shown how this problem can be solved using a novel subspace approach based on angles between subspaces. The method was demonstrated to have good performance, resulting in both accurate estimates of the spatial frequencies and the number of sources. Moreover, despite the introduction of compressed sensing it is possible to implement the algorithm using FFTs or root methods provided that a re-orthogonalization step is introduced. Interestingly, the results show, that the threshold behavior of the method changes with the use of compressed sensing, with the method still outperforming the ESPRIT algorithm.

\section{REFERENCES}

[1] R. O. Schmidt, "Multiple emitter location and signal parameter estimation," IEEE Trans. Antennas Propag., vol. 34(3), pp. 276-280, Mar. 1986.

[2] R. Roy and T. Kailath, "ESPRIT - estimation of signal parameters via rotational invariance techniques," IEEE Trans. Acoust., Speech, Signal Process., vol. 37(7), July 1989.

[3] G. Bienvenu, "Influence of the spatial coherence of the background noise on high resolution passive methods," in Proc. IEEE Int. Conf. Acoust., Speech, Signal Processing, 1979, pp. 306-309.

[4] D. Donoho, "Compressed sensing," IEEE Trans. Inf. Theory, vol. 52(4), pp. 1289-1306, apr 2006. 


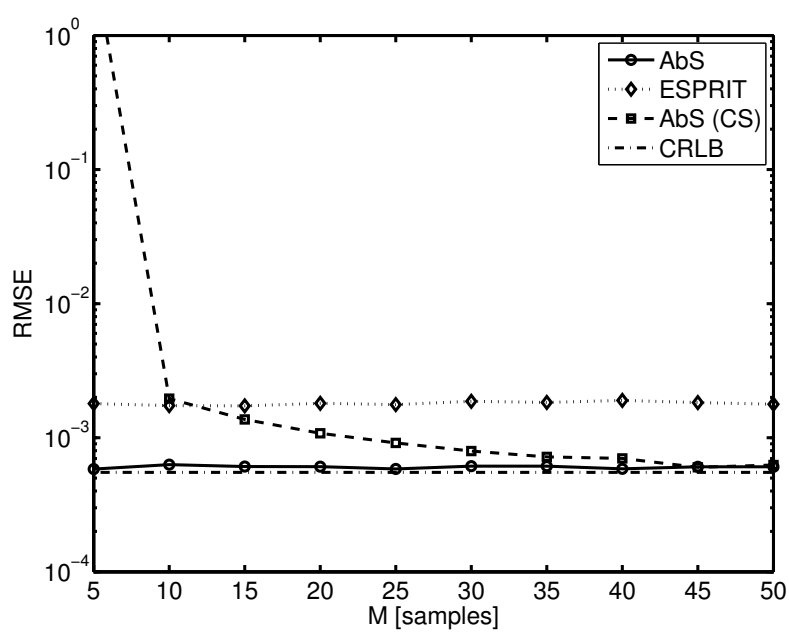

Fig. 3. RMSE as a function of measurements $M$.

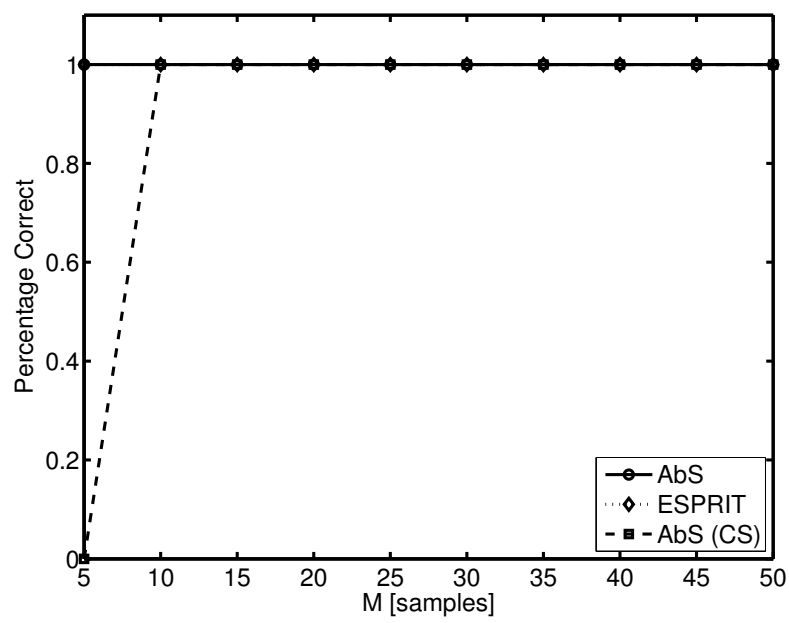

Fig. 4. Percentage of correctly estimated model orders as a function of the number of measurements $M$.

[5] E. Candés and M. Wakin, "An introduction to compressive sampling," IEEE Signal Process. Mag., vol. 25(2), pp. 21-30, Mar. 2008.

[6] D. Malioutov, M. Cetin, and A. S. Willsky, "A sparse reconstruction perspective for source localization with sensor arrays," IEEE Trans. Signal Process., vol. 53(8), pp. 3010-3022, Aug. 2005.

[7] A. C. Gübüz, J. H. McClellan, and V. Cevher, "A compressive beamforming method," in Proc. IEEE Int. Conf. Acoust., Speech, Signal Processing, 2008, pp. 2617-2620.

[8] V. Cevher, M. F. Duarte, and R. G. Baraniuk, "Distributed target localization via spatial sparsity," in Proc. European Signal Processing Conf., 2008.

[9] G. H. Golub and C. F. V. Loan, Matrix Computations, The Johns Hopkins University Press, 3rd edition, 1996.

[10] R. T. Behrens and L. L. Scharf, "Signal processing applications of oblique projection operators," IEEE Trans. Signal Process., vol. 42, no. 6, pp. 1413-1424, 1994.

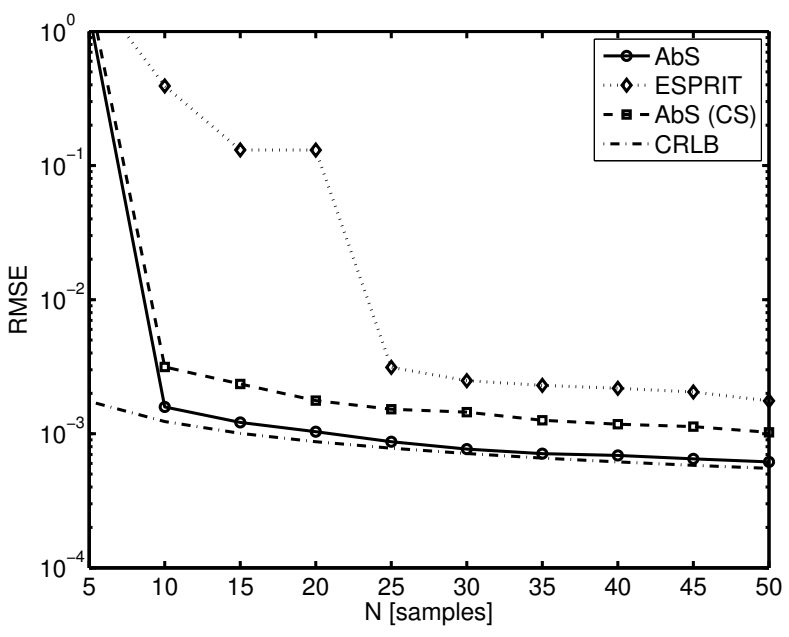

Fig. 5. RMSE as a function of the number of snapshots $N$.

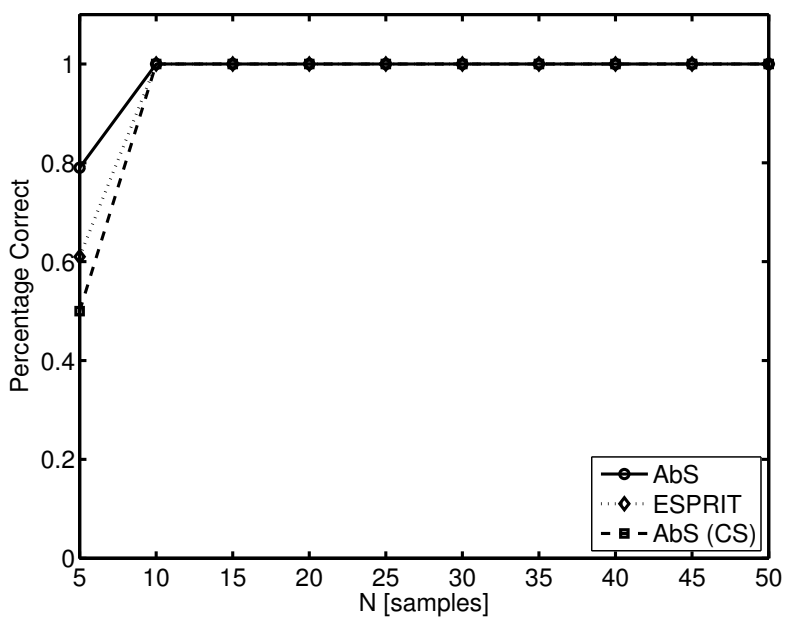

Fig. 6. Percentage of correctly estimated model orders as a function of the number of the snapshots $N$.

[11] M. G. Christensen, A. Jakobsson, and S. H. Jensen, "Sinusoidal order estimation using angles between subspaces," EURASIP J. on Advances in Signal Processing, pp. 1-11, 2009.

[12] R. Baraniuk, M. Davenport, R. Devore, and M. Wakin, "A simple proof of the restricted isometry property for random matrices," Constr. Approx, vol. 28(3), pp. 253-263, 2007.

[13] E. J. Candés, Y. C. Eldar, and D. Needell, "Compressed sensing with coherent and redundant dictionaries," May 2010, unpublished manuscript.

[14] S. C., D. L. Donoho, and M. A. Saunders, "Atomic decomposition by basis pursuit," SIAM J. Sci. Comput., vol. 20, pp. 33-61, 1996.

[15] R. Badeau, B. David, and G. Richard, "A new perturbation analysis for signal enumeration in rotational invariance techniques," IEEE Trans. Signal Process., vol. 54, no. 2, pp. 450458, Feb. 2006. 